\title{
An efficient approach to identify Ginkgo biloba cultivars by using random amplified polymorphic DNA markers with a manual cultivar identification diagram strategy
}

\author{
G.P. Li ${ }^{1}$, C.Q. Zhang ${ }^{2}$ and F.L. Cao ${ }^{1}$ \\ ${ }^{1}$ College of Forest Resources and Environment, Nanjing Forestry University, \\ Nanjing, China \\ ${ }^{2}$ College of Horticulture, Jinling Institute of Technology, Nanjing, China \\ Corresponding author: F.L. Cao \\ E-mail: samcaoster@gmail.com \\ Genet. Mol. Res. 12 (1): 175-182 (2013) \\ Received April 19, 2012 \\ Accepted November 8, 2012 \\ Published January 24, 2013 \\ DOI http://dx.doi.org/10.4238/2013.January.24.10
}

\begin{abstract}
Cultivar identification is a key step to avoid the formation of homonyms and synonyms of Ginkgo biloba. In this study, a new approach based on combinational utilization of polymorphic bands produced from 6 different random amplified polymorphic DNA (RAPD) primers was developed for identifying 42 Ginkgo cultivars, and a manual cultivar identification diagram that consisted of polymorphic bands produced from different RAPD primers was reported. To check the reliability and efficiency of the cultivar identification diagram, 5 randomly chosen cultivars were further tested, and the workability of the diagram was verified. This new approach will be very helpful for Ginkgo cultivar discrimination and protection, and will also be beneficial for the nursery industry for early identification of Ginkgo seedlings.
\end{abstract}

Key words: Ginkgo biloba; Cultivar identification; RAPD markers 


\section{INTRODUCTION}

Ginkgo (Ginkgo biloba L.) is one of the oldest living trees, and once had several species during the Lower Cretaceous period (Major, 1967). Currently, there is only a single surviving wild species, which is exclusively found in southeastern China. Despite this, its high value as medicine, food, timber, or ornament renders this tree worthy of cultivation throughout the world (Saevels and Corthout, 2005; Wang et al., 2006; Abad et al., 2010). In China alone, its cultivated areas have grown to approximately 400,000 hectares through 2011, and the value of its annual output was reported in the same year to be over 2.9 billion dollars. As necessary, cultivar exchanges in different regions and/or countries naturally become greatly important as cultivation efforts expand. However, since Ginkgo cultivars are often named based on a local name or vernacular names, homonyms, and synonyms are quite common (Zhong et al., 2009), which greatly affects cultivar exchanges and protection, and inhibits development of the Ginkgo industry.

Some classical approaches based on morphological or agronomic traits have been applied to distinguish cultivars in G. biloba (Christianson and Niklas, 2011), although the applications remain limited since the traits used by them, including leaf shape, fruit color, and plant height, are strongly related to environment and depend on copious observations in mature plants. Biochemical markers, including isozyme and allozyme markers, have also been applied to identify Ginko cultivars; however, their applications are also limited, as obtaining reliable results is only possible when the number of cultivars being tested is small. A perfect polymorphic marker is not easily obtained when distinguishing between many cultivars. In recent decades, molecular markers, including restriction fragment length polymorphism, random amplified polymorphic DNA (RAPD), simple sequence repeat, and others, have also been used in cultivar identification (Fan et al., 2004; Kuddus et al., 2002; Gong et al., 2008), which have the advantages of avoiding an environmental impact, not requiring copious observations in mature plants, and this approach can provide more polymorphic DNA bands. RAPD has more advantages than others in distinguishing the Ginkgo cultivars, since only little $a$ priori information, whether related to DNA sequence or related to enzymes, has to be obtained (Haussmann et al., 2002). Other methods more strongly depend on prior information. Primers used in RAPD are designed at random, and resultant polymorphic straps originate entirely from the variation of primer binding sites and DNA length differences between binding sites; thus, little a priori information is needed (Zhang et al., 2011).

Recently, a new strategy adapted to distinguish between many cultivars based on RAPD technology has been applied in grapevine, pears, plums, and peaches (Lin et al., 2011; Zhao et al., 2011), which provides a new potential approach for Ginkgo cultivar identification. This strategy begins with a presentation of effective primers for identifying the cultivar group, and then distinguishes each cultivar by combing polymorphic bands produced from different effective primers in a manual method. In this study, we evaluated the utility of this approach for the cultivar identification of Ginkgo. As a result, an effective method was established, and a manual cultivar identification diagram that could be used to direct cultivar discrimination was also reported. The current report presents the information required for identification of cultivar groups or a single cultivar, and provides a practical and theoretical basis for cultivar identification, genetic diversity analysis and the protection of new varieties through distinctness, uniformity and stability testing (Lu et al., 2009). 


\section{MATERIAL AND METHODS}

\section{Plant materials}

In our study, 42 G. biloba cultivars (Table 1) for cultivar identification and 5 for cultivar discrimination were used. All of the plant materials were obtained from the germplasm nursery of G. biloba, located in Pizhou, a city in Jiangsu, China.

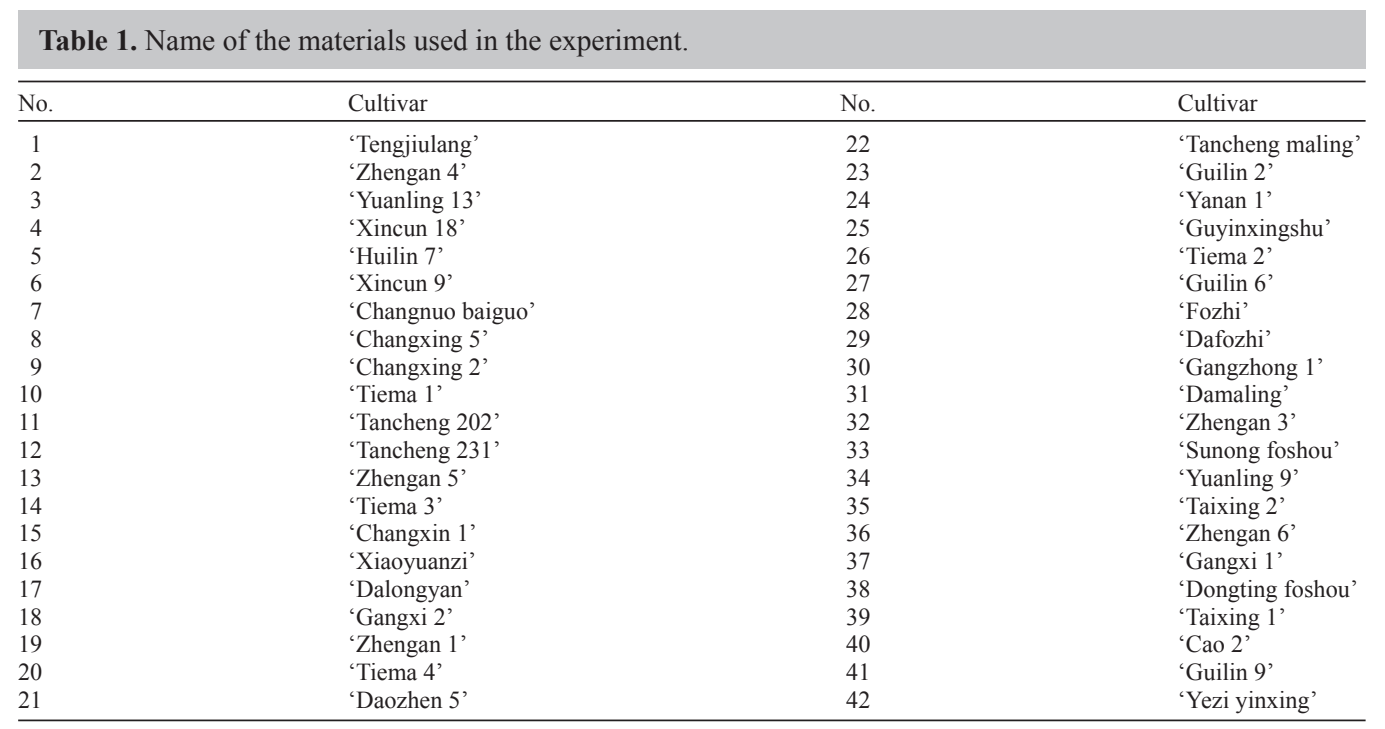

\section{Genomic DNA extraction}

To extract DNA, fresh young leaves of each cultivar were collected from a tree, flash frozen in liquid nitrogen, and stored at $-40^{\circ} \mathrm{C}$ for analysis. The modified cetyltrimethylammonium bromide method (Murray and Thompson, 1980) was used for DNA extraction.

Extracted DNA was further purified and checked for quality by electrophoresis on a $1 \%$ agarose gel, and diluted to a final concentration of $50 \mathrm{ng} / \mu \mathrm{L}$ with $1 \mathrm{X}$ TE buffer and stored at $-4^{\circ} \mathrm{C}$ until use.

\section{RAPD amplification}

RAPD reaction solutions consisted of $2.0 \mu \mathrm{L} 10 \mathrm{X}$ buffer, $1.2 \mu \mathrm{L} 25 \mathrm{mM} \mathrm{MgCl}_{2}, 1.6$ $\mu \mathrm{L} 2.5 \mathrm{mM}$ deoxyribonucleotide triphosphate, $1.6 \mu \mathrm{L} 1.0 \mu \mathrm{M}$ primer, $0.1 \mu \mathrm{L} 5 \mathrm{U} / \mu \mathrm{L} r T a q$ Polymerase Dynazyme, and $1 \mu \mathrm{L}$ genomic DNA, to a total volume of $20 \mu \mathrm{L}$. Amplification reactions were performed based on the standard protocol of Williams et al. (1990) with minor modifications, which were performed in an Eppendorf Authorized Thermal Cycler under the following program: an initial predenaturation step for $5 \mathrm{~min}$ at $94^{\circ} \mathrm{C}$, then 42 cycles each consisting of a denaturation step for $30 \mathrm{~s}$, an annealing step for $1 \mathrm{~min}$ at annealing temperature 
and an extension step for 2 min at $72^{\circ} \mathrm{C}$. Amplification was terminated by a final extension at $72^{\circ} \mathrm{C}$ for $10 \mathrm{~min}$. After amplification, the amplified DNA fragments were separated by gel electrophoresis on $1.3 \%$ agarose $(\mathrm{w} / \mathrm{v})$ in $1 \mathrm{X}$ Tris-acetate-ethylenediaminetetraacetic acid (0.04 M Tris-acetate, $0.001 \mathrm{M}$ ethylenediaminetetraacetic acid, $\mathrm{pH} 8.0$ ) buffer at $100 \mathrm{~V}$. The gels were stained with $0.5 \mu \mathrm{g} / \mathrm{mL}$ ethidium bromide and visualized under ultraviolet light for polymorphic bands from the cultivars.

\section{RESULTS}

\section{Quality of extracted DNA and optimization of RAPD conditions}

The extracted genomic DNA of each Ginkgo cultivar was checked by electrophoresis on a $1 \%$ agarose gel. The results showed that each sample had clear DNA bands (Figure 1), indicating the presence of genomic DNA.

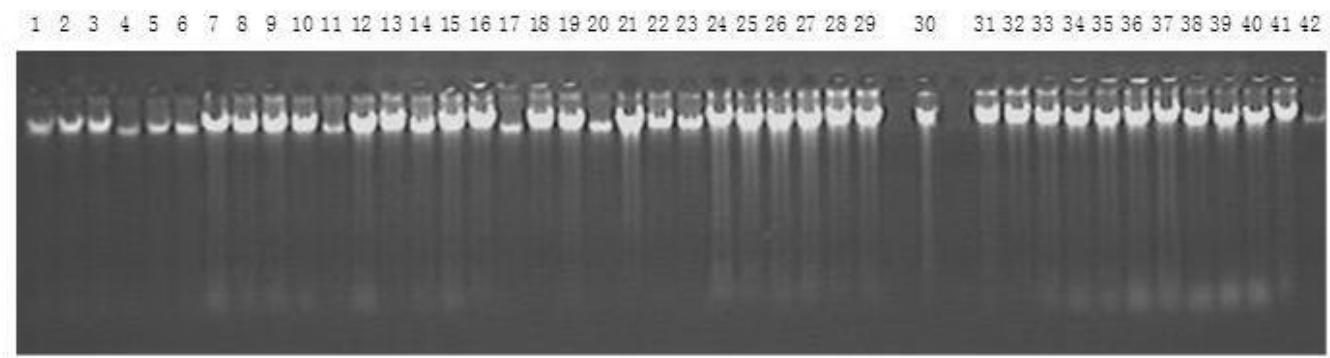

Figure 1. Check of extracted genomic DNA. Lanes 1 to $42=$ accession numbers of Ginkgo biloba cultivars listed in Table 1.

To further build a stable RAPD system with high reproducibility, 30 11-mer random primers were used for selection, and their annealing temperatures were also strictly screened based on the quality and yield of the band(s). Upon a primer producing clear, reproducible polymorphic bands when it was screened, it was utilized for the identification of Ginkgo cultivars. After this process was complete, 6 primers and their corresponding annealing temperatures were obtained (Table 2).

Table 2. Six primers chosen for identification of 42 Ginkgo biloba genotypes.

\begin{tabular}{llr}
\hline Primer & Nucleotide sequence $\left(5^{\prime}-3^{\prime}\right)$ & Annealing temperature $\left({ }^{\circ} \mathrm{C}\right)$ \\
\hline Y4 & GTTTCGCTCCT & 44.4 \\
Y6 & GTTTCGCTCCC & 42.8 \\
Y10 & CTGCTGGGACT & 44.4 \\
Y36 & AAGCCTCGTCC & 43.7 \\
Y42 & AGCGTCCTCCC & 45.1 \\
Y58 & ACCCCCGACTT & 43.7 \\
\hline
\end{tabular}

\section{Cultivar identification}

In order to build an effective method for cultivar identification of Ginkgo, we utilized 
a modified strategy from that of RAPD for the 42 collected cultivars. As a result, all 42 Ginkgo cultivars were successfully separated by the polymorphic straps produced from application of the above 6 primers, and a manual cultivar identification diagram was also constructed.

As shown in Figure 2, Primer Y4 is the first primer used to amplify the 42 G. biloba cultivars. The polymorphic bands with sizes of 1400 bp (Figure 2) were chosen to separate the 42 cultivars, and the presence and/or absence of the band (Figure 2) could classify these cultivars into 2 groups.

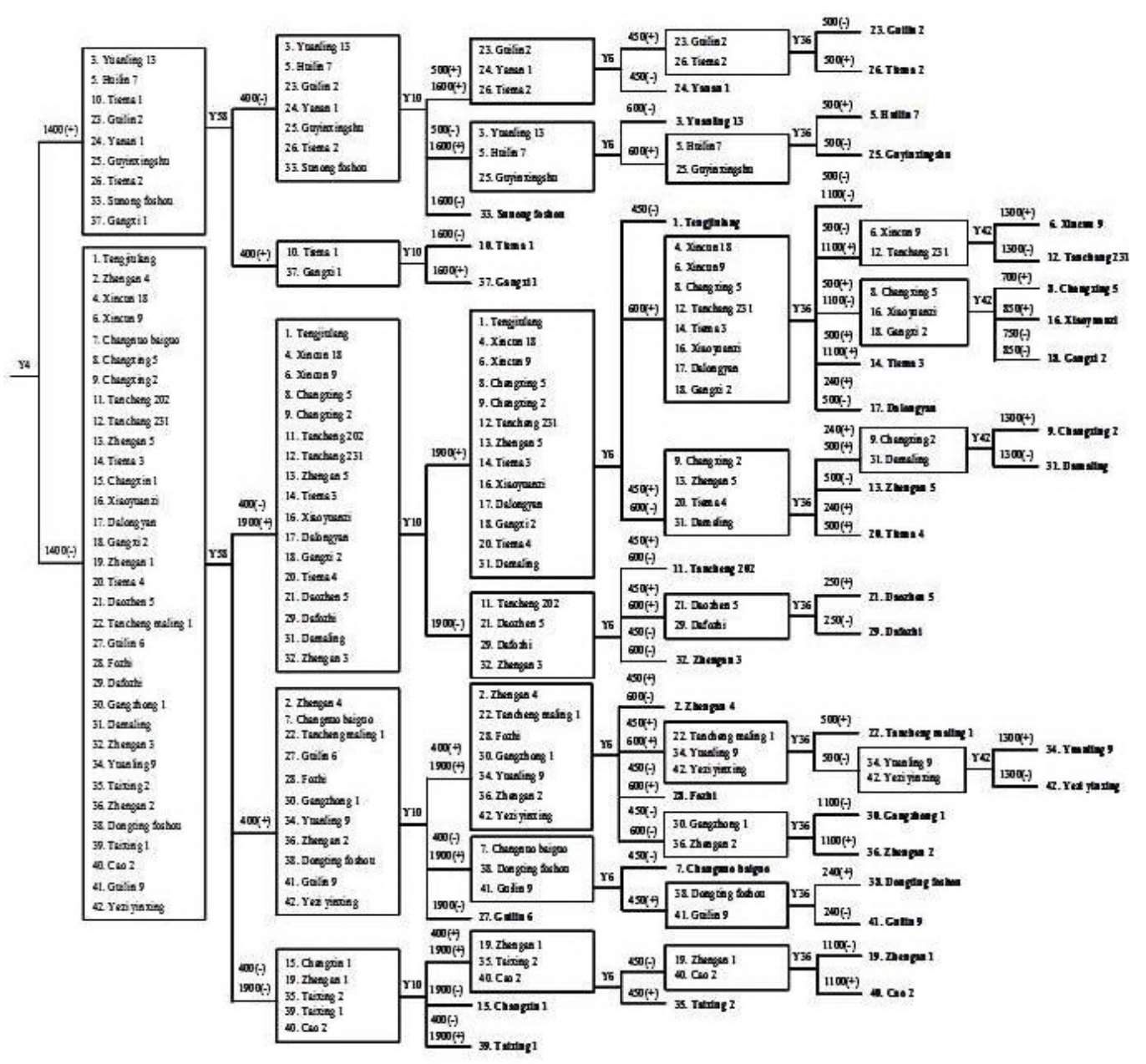

Figure 2. Manual cultivar identification diagram of 42 Ginkgo biloba cultivars by 6 primers. Y4, Y6, Y10, Y36, Y42, and Y58 are the names of primer sequences listed in Table 2. Numbers in front of $(+) /(-)$ indicate the size (in $\mathrm{bp)}$ of the polymorphic bands produced from application of primers Y4, Y6, Y10, Y36, Y42, or Y58. $(+)=$ presence of polymorphic band; $(-)=$ absence of polymorphic band.

The first group, including 9 G. biloba cultivars could be further separated into 2 subgroups based on the information regarding the presence and/or absence of a 400-bp sequence 
amplified by primer Y58 (Figure 3). Of the 2 subgroups, "Tiema 1" and "Gangxi 1" in the second subgroup were first identified as a single-cultivar group by primer Y10. The first subgroup, including 7 cultivars, was gradually separated by 3 primers (Y10, Y6, and Y36). The second group, including 33 cultivars separated by primer Y4, was also gradually separated by several primers, and the related information is mentioned in Figure 2. Finally, all of the $G$. biloba cultivars were successfully separated by using the 6 primers.

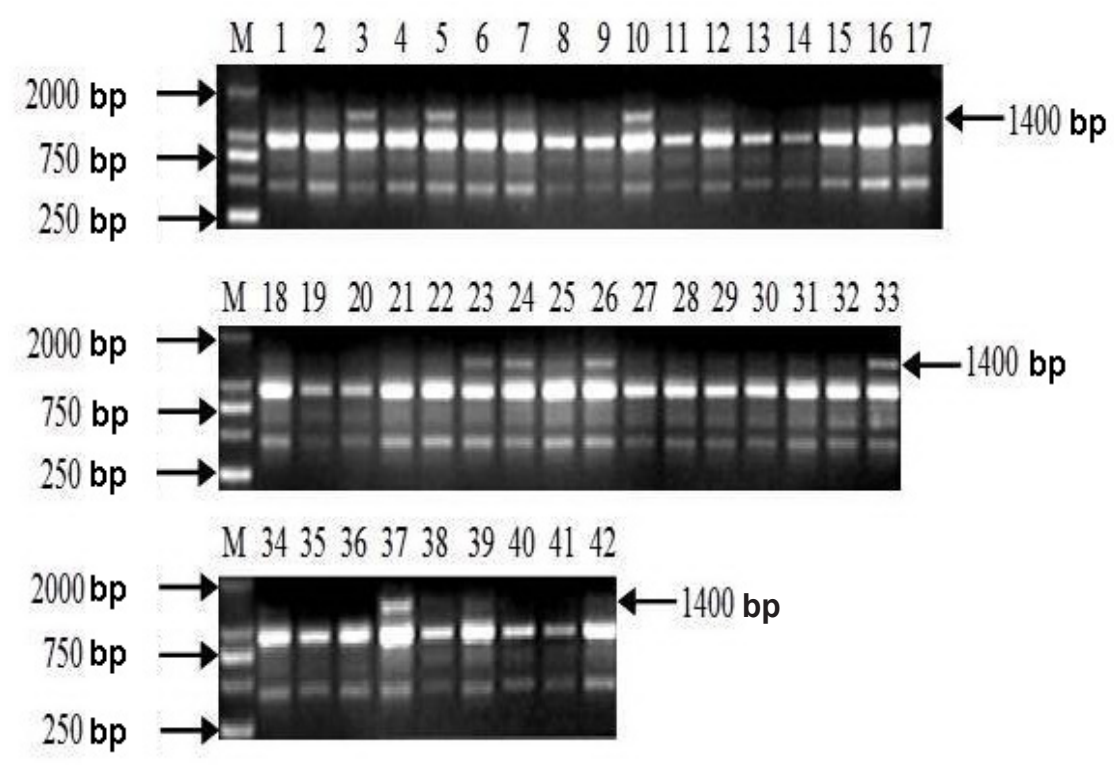

Figure 3. DNA banding patterns of 42 Ginkgo biloba cultivars amplified by primer Y4. Lane M= DL2000 plus DNA ladders; lanes 1 to 42 = accession numbers of Ginkgo biloba cultivars listed in Table 1.

\section{Application of the cultivar identification diagram on cultivar discrimination}

To further evaluate the workability of the manual cultivar identification diagram obtained above, 5 cultivars, randomly chosen from the germplasm nursery and claimed to be "Tengjiulang", "Zhengan 4", "Yuanling 13", "Xincun 18", and "Huilin 7", were used for cultivar discrimination.

From the location of the manual cultivar identification diagram (Figure 2), it was easy to find the primers (Y4, Y6, and Y58) used for separating these cultivars. Thus, we used these specific primers for cultivar discrimination.

Clearly, as illustrated in Figure 4, the PCR results of these cultivars using the corresponding primers were the same as those anticipated. The PCR results showed that 2 G. biloba cultivars ("Yuanling 13" and "Huilin 7") were first identified as a singlecultivar group by $\mathrm{Y} 4$ and $\mathrm{Y} 6$, the other 3 cultivars were separated by primers $\mathrm{Y} 4$, Y 58, and Y6. By this procedure, all 5 cultivars were successfully discriminated with the suitable combination of primers. Therefore, this test confirmed the availability, workability and efficiency of this diagram for use in the identification of G. biloba cultivars. 


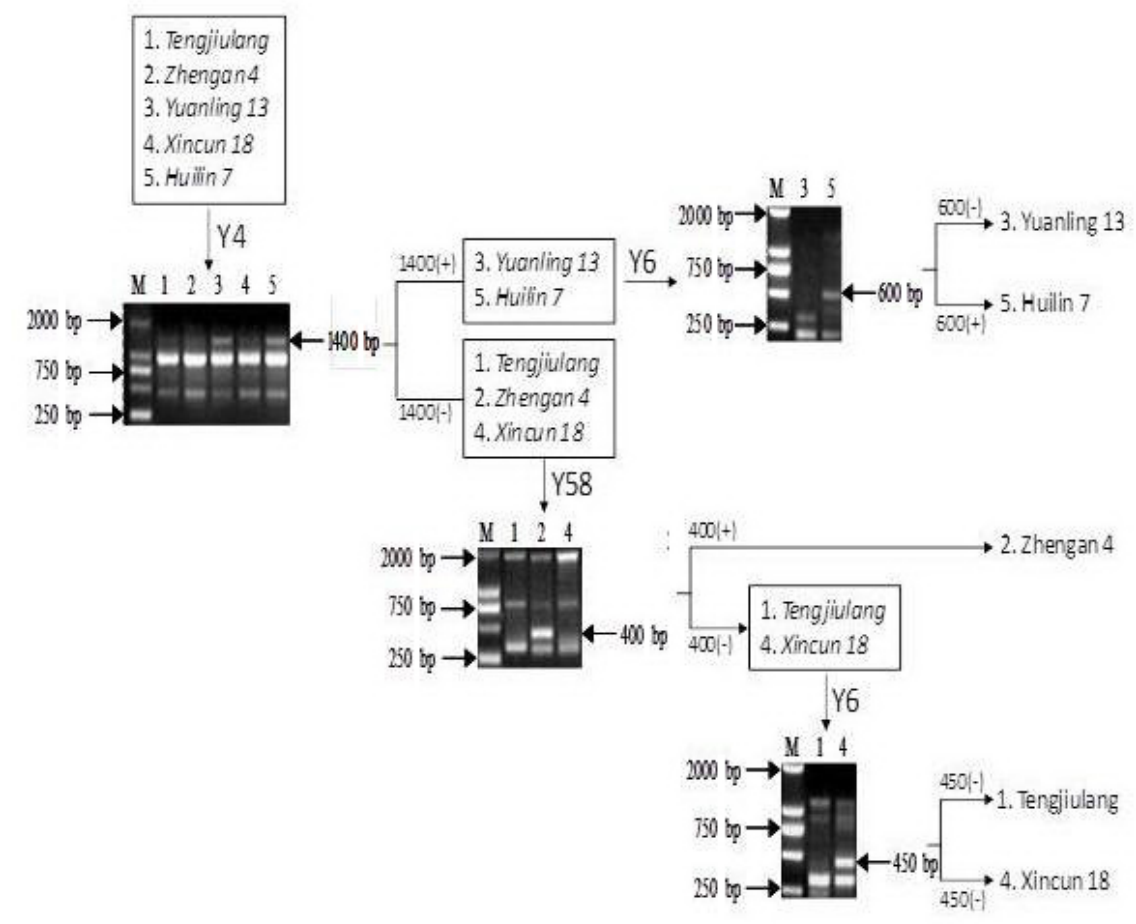

Figure 4. Discrimination of 5 tested Ginkgo biloba cultivars. Italic font in text box indicates the name of cultivar to be confirmed. In the DNA banding patterns, lane $M=$ DL2000 plus DNA ladders; lanes 1 to $5=$ accession numbers of Ginkgo biloba cultivars listed in text box. Y4, Y6, and Y58 are the names of primer sequences listed in Table 2.

\section{DISCUSSION}

Ginkgo is important in medicine, food, timber, and ornament, and its cultivated areas and annual output value have rapidly grown in recent years throughout the world. However, homonym and synonym frequently affected Ginkgo cultivar exchange and protection efforts (Zhong et al., 2009). China is a major Ginkgo-producing country, thus cultivar identification has become imperative for plant scientists, with the purpose of servicing agricultural growth and variety protection. However, very little research has been performed on developing efficient cultivar identification strategies. In this study, 6 novel RAPD primers producing clear, reproducible polymorphic bands were developed for distinguishing G. biloba cultivars, and a manual cultivar identification diagram based on different combinations of polymorphic bands exemplified by these 6 primers was built for identification of 42 Ginkgo cultivars.

Compared to other methods, this cultivar identification diagram renders DNA markers sufficiently workable in Ginkgo cultivar identification. It is useful for choosing specific primers that can be used to discriminate between G. biloba cultivars. In general, any 2 cultivars can be identified with a single RAPD primer. For cases in which more G. biloba cultivars are confused in practice, the set of 6 primers can be used to run the DNA samples of the new cultivars, and the PCR banding patterns identify the new cultivars. In some cases, the new cultivars might not contain the 42 cultivars included in this diagram, although it can also separate them by further 
introducing new primers and positioning them on the diagram to form a more ideal guided diagram. The cultivar identification diagram is an open diagram, and allows users to add a new cultivar to it, which is very helpful for genetic resource conservation and utilization, plant variety protection and cultivar exchanges (Lin et al., 2011; Zhao et al., 2011).

The strategy used for building the Ginkgo cultivar identification diagram does not require much more study and time to be spent for cultivar identification; in addition, genetic linkage maps or DNA sequence data are not required, in theory. This strategy will be more widely applied in the future. However, while research is being conducted, the manual cultivar identification diagram might be difficult to meet a fast query. Thus, in our subsequent study we will aim to build a query database by storing the information contained in the cultivar identification diagram and make it available to scientists (Zhang et al., 2011) and other users throughout the world.

\section{ACKNOWLEDGMENTS}

Research supported by the National Science Foundation of China (\#31170627, \#31171273) and the Doctoral Fund of Education Ministry (\#011010197).

\section{REFERENCES}

Abad MJ, Bedoya LM and Bermejo P (2010). An update on drug interactions with the herbal medicine Ginkgo biloba. Curr. Drug Metab. 11: 171-181.

Christianson ML and Niklas KJ (2011). Patterns of diversity in leaves from canopies of Ginkgo biloba are revealed using Specific Leaf Area as a morphological character. Am. J. Bot. 98: 1068-1076.

Fan XX, Shen L, Zhang X, Chen XY, et al. (2004). Assessing genetic diversity of Ginkgo biloba 1. (Ginkgoaceae) populations from China by RAPD markers. Biochem. Genet. 42: 269-278.

Gong W, Zeng Z, Chen YY, Chen C, et al. (2008). Glacial refugia of Ginkgo biloba and human impact on its genetic diversity: evidence from chloroplast DNA. J. Integr. Plant Biol. 50: 368-374.

Haussmann G, Hess E, Seetharama N, Welz G, et al. (2002). Construction of a combined sorghum linkage map from two recombinant inbred populations using AFLP, SSR, RFLP, and RAPD markers, and comparison with other sorghum maps. Theor. Appl. Genet. 105: 629-637.

Kuddus RH, Kuddus NN and Dvorchik I (2002). DNA polymorphism in the living fossil Ginkgo biloba from the eastern United States. Genome 45: 8-12.

Lin J, Wang XC, Chang YH and Fang JG (2011). Development of a novel and efficient strategy for practical identification of Pyrus spp (Rosaceae) cultivars using RAPD fingerprints. Genet. Mol. Res. 10: 932-942.

Lu X, Liu L, Gong Y, Zhao L, et al. (2009). Cultivar identification and genetic diversity analysis of broccoli and its related species with RAPD and ISSR markers. Sci. Hortic. 122: 645-648.

Major RT (1967). The ginkgo, the most ancient living tree. The resistance of Ginkgo biloba L. to pests accounts in part for the longevity of this species. Science 157: 1270-1273.

Murray MG and Thompson WF (1980). Rapid isolation of high molecular weight plant DNA. Nucleic Acids Res. 8: 43214325.

Saevels J and Corthout J (2005). Ginkgo biloba medicines and food supplements on the Belgian market: a comparative study. J. Pharm. Belg. 60: 129-134.

Wang L, Xing SY, Yang KQ, Wang ZH, et al. (2006). Genetic relationships of ornamental cultivars of Ginkgo biloba analyzed by AFLP techniques. Yi Chuan Xue Bao 33: 1020-1026.

Williams JG, Kubelik AR, Livak KJ, Rafalski JA, et al. (1990). DNA polymorphisms amplified by arbitrary primers are useful as genetic markers. Nucleic Acids Res. 18: 6531-6535.

Zhang C, Wang J, Hua X, Fang J, et al. (2011). A mutation degree model for the identification of transcriptional regulatory elements. BMC Bioinformatics 12: 262.

Zhao MZ, Zhang YP, Wu WM, Wang C, et al. (2011). A new strategy for complete identification of 69 grapevine cultivars using random amplified polymorphic DNA (RAPD) markers. Afr. J. Plant Sci. 5: 273-280.

Zhong XK, Li DC and Jiang JG (2009). Identification and quality control of Chinese medicine based on the fingerprint techniques. Curr. Med. Chem. 16: 3064-3075. 